\title{
Relatos de Experiencia en Torno
a la Constitución de
Subjetividad Política Experiencia en Torno
a la Constitución de
Subjetividad Política Experiencia en Torno
a la Constitución de
Subjetividad Política
}

\footnotetext{
${ }^{1}$ Institución Educativa Técnica Agropecuaria San Rafael, Rondón angelica.carob@gmail.com

Como citar: Caro, A. (2021). Relatos de Experiencia en Torno a la Constitución de Subjetividad Política. Educación y Ciencia, (25), e12631. https://doi.org/10.19053/0120-7105. eyc.2021.25.e12631
}

\author{
Angélica Caro Bermúdez ${ }^{1}$
}

\section{Resumen}

El artículo presenta avances del proyecto "Subjetividad política: relatos de experiencia de una maestra de educación preescolar en el contexto de una escuela multigrado", el cual se interesa por reconocer elementos que configuran la subjetividad política de una maestra de escuela multigrado a través de un ejercicio investigativo y formativo de escritura narrativa. El trabajo se desarrolló desde un enfoque cualitativo de tipo hermenéutico-narrativo. Desde la perspectiva de la investigación narrativa, se asumió la escritura como un ejercicio de formación que posibilita construir nuevos horizontes de sentido en torno a las prácticas.

El texto presenta avances de la investigación a partir de la elaboración y relaboración de relatos autobiográficos en torno a la práctica pedagógica. Se estructuran dos categorías de análisis: lo político en el cuidado de la vida y el distanciamiento crítico frente a formas preestablecidas del ser docente. Las cuales posibilitaron reflexionar sobre cómo los procesos de formación permiten la configuración de la subjetividad política a través de narrativas de experiencias educativas.

Palabras clave: maestro, subjetividad política, experiencia, narración, formación. 


\title{
Stoires of Experience On The Constitution Of Political Subjectivity
}

\begin{abstract}
The article presents advances of the project Juan, which aim was to recognize elements that shape the political subjectivity of a multi-grade school teacher through an investigative and formative exercise in narrative writing. The work used a qualitative approach of hermeneutics-narrative type. From the perspective of narrative research, writing was an exercise of training that made it possible to build new horizons of meaning around practices.

It presents advances of pedagogical practice from the elaboration and reworking of autobiographical stories. Two categories of analysis are structured: the political in the care of life and the critical distancing pre-established forms of being a teacher. Which made it possible to reflect on how training processes allow the configuration of political subjectivity through narratives of educational experiences.
\end{abstract}

Keywords: teacher, political subjectivity, experience, narration, training.

\section{Introducción}

La subjetividad política se constituye como categoría de estudio en el proyecto de investigación "Subjetividad política: relatos de experiencia de una maestra de educación preescolar en el contexto de una escuela multigrado", el cual se interesa por reconocer los sentidos de lo político en el desarrollo de la práctica pedagógica de una maestra de escuela multigrado. Siguiendo a Itaí (2012) para Miriam Kriger "somos sujetos políticos cuando nos percibimos a nosotros mismos, reflexivamente, como agentes sociales con conciencia de nuestra identidad histórica, que apostamos a tomar decisiones a futuro y nos sentimos responsables de la dimensión política de nuestras acciones" ( p. 1). Por esta razón, cuando los maestros se cuestionan sobre la pertinencia del proceso escolar, adquieren una subjetividad crítica que les permite reconocerse como sujetos transformadores y creadores, y los dota de claridad respecto al trabajo que realizan a diario, el cual, va más allá de la transmisión de conocimientos y la reproducción ciega, casi sin darse cuenta, de un sistema autoritario, patriarcal, desigual y excluyente. Este reconocimiento de sí mismos ayuda a los sujetos a construir su subjetividad, la cual le permite comprender que:

Somos humanamente diferentes, irrepetibles, pero venimos de historias compartidas bio-filo-epi y ontogenéticamente, lo cual nos hace comunes a la especie humana, pero se hace específica en cada uno de acuerdo a contextos, culturas, historias, intereses, visibles en los lenguajes y habilidades que desarrollemos desde donde construimos nuestra subjetividad, que siempre está rehaciéndose (Mejía, 2020, p. 46).

Al hablar de subjetividad política es preciso realizar un ejercicio de análisis propio 
que permita reconocer, construir y transformar nuestro mundo. En este proceso, el campo de la experiencia toma vital importancia porque es a partir de lo que nos sucede y de la elaboración que hacemos de estos sucesos que podemos generar experiencias para formarnos y transformarnos, a través del cuidar de sí mismos y también de las relaciones cotidianas con las personas con que interactuamos o hacen parte de nuestra vida. Estas experiencias se van dando a medida que las vamos reelaborando y una forma de hacerlo es a través del lenguaje: hablando, escribiendo, narrando y contando lo que sucede, de esta manera constituimos una subjetividad crítica que permite configurarnos a partir de lo que nos pasa. Al respecto Passeggi (2011) considera que:

Las narrativas autobiográficas como dispositivos de formación y auto-reflexión, en la perspectiva freiriana, no se limitan, jamás, a una visión intimista de mí, sino que de revelan en un proceso de objetivación, de socialización, que transforman una narrativa privada en una narrativa civil, en la cual el narrador, al buscar comprehender su historicidad, hace uso de sus márgenes de maniobra reales para insertar su acción en la Historia (p. 9).

Teniendo en cuenta lo anterior, en el marco de esta investigación se elaboraron y reelaboraron relatos que permitieron resignificar la experiencia pedagógica de una maestra a partir de la reconstrucción de sus trayectorias de formación. Estos relatos se realizaron siguiendo preguntas orientadoras que posibilitaron un ejercicio de escritura narrativa, con el fin de dar respuesta a la pregunta ¿cuáles son los sentidos de lo político que se han ido configurando a lo largo de la experiencia de formación de una maestra de educación preescolar en el contexto de su trayectoria de vida?

\section{Experiencia, Formación y Transformación}

La experiencia es una categoría fundamental a trabajar en un proceso de constitución de subjetividades políticas, puesto que permite que los sujetos analicen sus propias prácticas y a partir de ellas puedan reelaborarlas y generar nuevos saberes. La experiencia se da a partir de los significados, el análisis, la interpretación y la reelaboración que cada ser humano da a las cosas que le ocurren, por eso, un mismo acontecimiento genera una experiencia diferente en cada persona que lo vivenció, porque cada uno lo asimila de manera particular de acuerdo a vivencias anteriores. Para Larrosa (2006) la experiencia es:

Un acontecimiento o, dicho de otro modo, el pasar de algo que no soy yo. Y "algo que no soy yo" significa también algo que no depende de mí, que no es una proyección de mí mismo, que no es el resultado de mis palabras, ni de mis ideas, ni de mis representaciones, ni de mis sentimientos, ni de mis proyectos, ni de mis intenciones, es algo que no depende ni de mi saber, ni de mi poder, ni de mi voluntad. "Que no soy yo" significa que es "otra cosa que yo", otra cosa que no es lo que yo digo, lo que yo sé, lo que yo siento, lo que yo pienso, 
lo que yo anticipo, lo que yo puedo, lo que yo quiero (p. 2).

Para Larrosa (2006), la experiencia está conformada por lo que él denomina como "principios", uno de los cuales es la exterioridad. Puesto que los eventos que le suceden a un sujeto no dependen de él, sino que son sucesos que vienen del exterior y cada uno se encarga de darle un significado diferente según las situaciones a las que ha tenido que enfrentarse, estas experiencias se van encargando de construir la subjetividad de cada persona. En este principio también son indispensables las relaciones sociales y los acontecimientos que se van dando a través de la interacción con las demás personas. En palabras de Larrosa (2006):

No hay experiencia, por tanto, sin la aparición de un alguien, o de un algo, o de un eso, de un acontecimiento, en definitiva, que es exterior a mí, extranjero a mí, extraño a mí, que esta fuera de mi mismo, que no pertenece a mi lugar, que no está en el lugar que yo le doy, que esta fuera de lugar (p. 3).

Un segundo principio es el de reflexividad, según el cual, para que la experiencia tenga lugar o se pueda dar se necesita de un sujeto, que le pasa un acontecimiento, quien a partir de sus vivencias se encarga de dotar de significado esos sucesos y de convertirlos en experiencias. Por esto se debe considerar que:

La experiencia es un movimiento de ida y vuelta. Un movimiento de ida porque la experiencia supone un movimiento de exteriorización, de salida de mí mismo, de salida hacia fuera, un movimiento que va al encuentro con eso que pasa, al encuentro con el acontecimiento. Y un movimiento de vuelta porque la experiencia supone que el acontecimiento me afecta a mí, que tiene efectos en mí, en lo que yo soy, en lo que yo pienso, en lo que yo siento, en lo que yo sé, en lo que yo quiero, etc. (Larrosa, 2006, p. 4).

Los últimos principios a trabajar aquí son los de formación y transformación, puesto que cada acontecimiento que ha sido convertido en experiencia contribuye a formar o transformar la subjetividad de los sujetos, por ello, no hay experiencia que no forme, que no eduque y que no ayude a transformar las ideas, pensamientos y acciones de las personas. Para Passeggi (2011) "la formación cuando adopta la mirada reflexiva sobre la experiencia vivida, en ningún momento debe ser entendida como una (trans)formación sin crisis" (p. 7), por esta razón es importante entender que no solo nos formamos gracias a los acontecimientos que consideramos como buenos, sino que, al reflexionar sobre los sucesos negativos es posible encontrar en ellos elementos que le permiten al sujeto transformarse.

\section{Narrar la Experiencia}

En la subjetividad política la experiencia es fundamental porque ayuda a reelaborar las vivencias que le suceden a diario a los sujetos. A los maestros les permite reflexionar sobre su práctica pedagógica para poder transformarla, en la medida que 
su subjetividad también se va transformando. El lenguaje resulta de vital importancia en la experiencia, porque permite narrar, contar, expresar y dar a conocer las vivencias que han permitido a los maestros constituir su subjetividad.

En el desarrollo de un proyecto investigativo, el narrar las experiencias permite al investigador ser el actor principal del proceso, es la voz del investigador la que se pone al descubierto y en la medida que va elaborando y reelaborando los relatos, las vivencias que se están narrando van adquiriendo otros sentidos, lo que posibilita que el sujeto se transforme. Al respecto Passeggi (2011) menciona:

Las narrativas autobiográficas como dispositivos de formación y auto-reflexión, en la perspectiva freiriana, no se limitan, jamás, a una visión intimista de mí, sino que de revelan en un proceso de objetivación, de socialización, que transforman una narrativa privada en una narrativa civil, en la cual el narrador, al buscar comprehender su historicidad, hace uso de sus márgenes de maniobra reales para insertar su acción en la Historia (p.9).

En los procesos investigativos con narrativas autobiográficas no solo se trata de contar la vida del investigador, sino de analizar las experiencias que han permitido que se forme como tal. Por eso es importante poder entrar en diálogo con los autores que se leen, poder reflexionar sobre todos y cada uno de los sucesos que influyen en la vida del investigador, y ver de qué manera el contexto social y las relaciones con los demás aportan a la constitución de la subjetividad del investigador.

\section{Investigación Biográfico Narrativa}

Esta investigación se trabajó bajo el enfoque hermenéutico porque este enfoque se centra comprender en interpretar los fenómenos humanos, haciendo del sujeto investigador el actor principal en el proceso investigativo, dando importancia al lenguaje y la construcción de saberes.

De acuerdo a Foucault (1994 [1982]) la cuestión del sujeto y del conocimiento del sujeto ha sido planteada bajo "la fórmula del Oráculo de Delfos: conócete a ti mismo. Pero, en realidad, esta fórmula de conócete a ti mismo va acompañada siempre, por otra parte, de otra exigencia: ocúpate de ti mismo" (p. 33). Desde este enfoque se plantea la necesidad de analizarse a sí mismo para poder transformase. Siguiendo tal principio, este estudio usó una metodología biográfico-narrativa que permitió a una maestra de educación preescolar elaborar y reelaborar relatos con sus vivencias, para poder reflexionar en torno al desarrollo de sus prácticas pedagógicas. La hermenéutica narrativa permite la comprensión de la complejidad psicológica de las narraciones que los individuos hacen de los conflictos y los dilemas de sus vidas, por ello, es un enfoque subjetivo y abierto a constantes cambios y transformaciones (Bolívar, citado en Africano, 2018). Las categorías que se generan fruto del análisis de los relatos, son producto de la integración narrativa que articula diversas experiencias. 
La investigación se realizó teniendo en cuenta cuatro momentos metodológicos:

1. Contextualización: A través de la elaboración de diarios de campo, la maestra investigadora registró sus narraciones relacionadas con vivencias diarias que permitieron contextualizar su práctica pedagógica.

2. Problematización: En este momento se construyó el distanciamiento con respecto a las prácticas pedagógicas que reproducen ideales sobre el deber ser del maestro, olvidando los contextos y los sujetos participes de la investigación.

3. Narración: A partir de la elaboración y reelaboración de relatos de experiencia por parte de la maestra, se reconocieron los aspectos que permitieron la configuración de su subjetividad política.

4. Resignificación: Mediante un proceso de lectura y reelaboración de relatos narrativos, se buscó reconocer nuevos saberes que permitieron a la maestra resignificar su práctica pedagógica.

\section{Análisis: Trayectorias de Formación}

\section{Lo Político en el Cuidado de la Vida}

Mi infancia transcurrió en la vereda Soconzaque, occidente del municipio de Boyacá, ubicada en el departamento que lleva el mismo nombre, en medio de la tranquilidad del campo y la algarabía de los niños del jardín infantil que tenía mi mamá en casa. Quizá ese fue el primer acercamiento a niños que no hacían parte de mi familia. A partir de eso pude darme cuenta de que los vínculos sanguíneos no son indispensables para sentir afecto por otras personas.

Aunque en la escuela me divertía con mis compañeras, siempre esperaba la hora de la salida para ir a mi casa a jugar y compartir con los niños del jardín. Esta rutina se vio interrumpida con mi paso al bachillerato porque la jornada académica era más extensa y tenía que desplazarme de la vereda al pueblo, y al llegar a casa solo podía ver como ellos se despedían alegremente de mi mamá prometiendo regresar al día siguiente. Quizá lo que más extrañaba de los niños era su alegría, espontaneidad para decir las cosas y su forma de ver el mundo.

Paralelo a esto, crecí en medio de la dureza de las labores del campo y la desigualdad en torno al rol tan marcado de la mujer con respecto al hombre. Aunque en mi hogar mi mamá tenía un trabajo estable, en cuanto terminaba de trabajar debía hacerse cargo de las labores de la casa, incluso cuando mi papá no tenía trabajo. Podía darme cuenta que en otros hogares el trabajo de la mujer era aún mayor, se hacían cargo de cocinar, alistar a los niños para la escuela y el jardín, ordenar, organizar la casa, cocinar y llevar la comida para los obreros, ayudar a trabajar hasta la hora de recoger a los niños, recoger leña, regresar a ordeñar y hacer la comida. Estas son solo algunas de las múltiples tareas que las mujeres de mi comunidad realizaban a diario. 
Con esto no quiero desconocer las labores que los hombres realizan en el campo, por el contrario, el trabajo del campo es bastante pesado: trabajar al sol y al agua sin ninguna garantía laboral y depender económicamente de factores externos... (buena cosecha, precios favorables, etc.), pero podía ver cómo las mujeres dependían del dinero que los hombres quisieran aportar para el hogar y no para ellas, y muchas veces era el dinero que sobraba luego que ellos pagaran obreros y se gastaran otro tanto tomando.

Adicionalmente, crecí viendo el poco reconocimiento que tenían las labores realizadas por las mujeres, por ejemplo, aunque mi mamá hacía tanto labores del campo como del hogar, para mi papá era como si ella no hiciera nada, incluso, la cuestionaba cuando ella manifestaba estar cansada. Aun así, mi mamá siempre ha estado muy pendiente de nosotras (somos cuatro hermanas) en todos los aspectos y desde hace unos años he podido darme cuenta de lo mucho que mi mamá hace, no solo por nosotras, sino también por las personas que la rodean, y en general por todos los que la necesiten. Esa valentía, fuerza y generosidad con la que ella se desenvuelve en su diario vivir ha hecho que considere esas prácticas de cuidado, presentes en mi formación, desde un sentido político.

Sin embargo, es preciso reconocer que no siempre ha sido así. Por algún tiempo quise incluso tomar distancia de ese tipo de prácticas pensando que, en lugar de favorecer mi formación política, le restaba posibilidades. De cierta manera, pensaba que las labores de cuidado hacían parte del sometimiento de las mujeres a una cultura patriarcal. Es ahora que intento hacer una reescritura de mis trayectorias de formación, que logro reconocer que el cuidado fue justamente lo que sostuvo no solamente mi vida, sino la de mi familia aún en circunstancias muy difíciles.

El ejercicio de narrar me ha permitido cuestionar valores y estructuras societales hegemónicas, lo cual, sin duda, tiene que ver con lo político, pero en medio de ese cuestionamiento, afirmar la posibilidad de otras formas de vida en donde la coexistencia en el cuidado de la vida sea a la vez una responsabilidad de todos y de cada uno, delinea lo político como un ejercicio vital, una práctica de vida que permite no solo la producción sino la reproducción de formas diversas de existencia.

En este sentido, he tenido un interesante encuentro con los feminismos, en tanto movimientos sociales y políticos que en sus diferentes vertientes no solo han cuestionado las estructuras del poder patriarcal, sino que, además, han señalado la posibilidad de encarnar formas de vida en las que la propia existencia se asuma como un permanente ejercicio de crítica, construcción y sostenimiento de otras formas de vida basadas en la coexistencia.

Este acercamiento me permitió entender que el sostenimiento de la vida tiene lugar gracias a prácticas de cuidado en las que está presente el sentido de lo colectivo, pero también, que las prácticas de cuidado afirman otra manera de asumir la propia 
existencia desde el vínculo, el afecto y la construcción de proyectos comunes. También ha hecho que reconozca de forma más clara el sentido político del cuidado y deje de pensar en que ellas (mi mamá y abuelita) por estar pendientes de los demás, no lo están de ellas. Por el contrario, he podido observar que ellas asumen el cuidado como una práctica afirmativa y colectiva que configura una manera diferente de habitar el mundo.

Durante mucho tiempo le resté importancia o no reconocí el valor de las prácticas que ellas realizaban, pero ahora puedo hacer otro tipo de lectura y me doy cuenta de que no es nada fácil; si cuidar de uno mismo requiere de mucha responsabilidad y dedicación, dedicarse al cuidado de los demás requiere un esfuerzo aún mayor. Con esto no quiero desconocer las luchas por la corresponsabilidad en las labores de cuidado, ni tampoco, las condiciones de desigualdad en que las mujeres tienen que ejercer dichas labores. Pero sí, reconocer su valor político dentro de las prácticas formativas que permitan constituir sujetos comprometidos con la sostenibilidad de la vida, para Castro (2018):

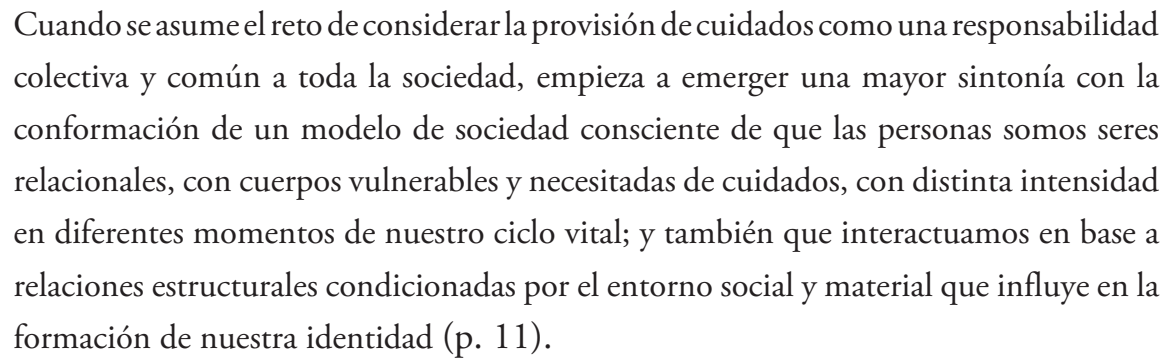

Entender las prácticas de cuidado como una responsabilidad de todos permite reivindicar tales prácticas y reconocer su valor en el funcionamiento de las sociedades, lo cual no solo atañe a las mujeres, sino que es una responsabilidad compartida. Comprender estos aspectos posibilita ubicar la vida en el centro del sistema social (Sáenz, 2018).

\section{Distanciamiento Critico con Formas Preestablecidas del Ser Docente}

El proceso de reconstrucción de mis trayectorias de formación fue bastante complejo, pues me exigió una profunda reflexión desde el pensar narrativamente la vida. En uno de mis primeros relatos de experiencia expresaba mi preocupación respecto a los contenidos, a trabajar con los estudiantes en la escuela rural en la que actualmente soy maestra. Relataba mi inquietud por los contenidos y la importancia de que mis estudiantes pudieran tener un buen desempeño en las evaluaciones, sobre todo las estatales, pues durante largo tiempo consideré que eso les iba a garantizar el acceso a la universidad y con esto, un futuro mejor.

Un día al hablar con mi tutora, luego de la lectura del relato, ella me preguntó porque pensaba que la vida en la ciudad era mejor que en el campo. Estuve pensando bastante tiempo esa pregunta; porqué pensaba eso, y me devolví a mi infancia. Como 
ya lo mencioné, yo nací y crecí en el campo y, generalmente, al hablar con mis primas, que estudiaban en la ciudad, podía ver como ellas comentaban de muchas cosas que conocían y que sabían, y yo solo podía sentirme ignorante al lado de ellas. Por lo que, cada vez comencé a hablar menos por miedo a decir cosas absurdas o simplemente porque no tenía nada que decir con respecto a las conversaciones que ellas tenían sobre su vida en la ciudad.

Por otro lado, sentía que mis tías, las hermanas de mi mamá, que también vivían en la ciudad, tenían mejores oportunidades laborales y económicas. Cuando viajaban para el campo mi mamá estaba pendiente de ayudarles en todo lo que necesitaban, e incluso mi mamá hacia cosas que ellas podían hacer. Sentía que no nos consideraban como iguales y nos trataban diferente a otros familiares. Pensaba que era por vivir en el campo y no tener los conocimientos que ellas habían adquirido. Comencé a cultivar la idea que estudiar y tener una carrera profesional iba a ser suficiente para que nos trataran diferente. Y no es que necesitara la aprobación de ellas, pero no me gustaba la relación que tenían con nosotros.

Por estas y otras razones, sin darme cuenta comencé a hacer de las calificaciones altas mi prioridad; sentía que eso podía garantizarme un mejor futuro. Siempre me comparaba con mis primas y por más que me esforzara nunca me sentía lo suficientemente buena frente a ellas. Cada vez más me alejaba de los demás y me sentía inferior a los demás. Me esforzaba en estudiar, en aprender muchas cosas para poder compartir con otros sin sentirme menos, pero por más que me esforzaba en memorizar información, al final terminaba olvidándola y, por lo tanto, evitando las conversaciones con los demás.

La estadía en la universidad hasta cierto punto fue difícil por la misma razón: eran muchísimas personas. Asimilar esto fue bastante difícil porque en la vereda éramos pocas personas y todos nos conocíamos. Este foco de atención en el ascenso social que pudiera darme la educación, me desvío del interés por las relaciones, tan fundamentales en las prácticas comunitarias. Iniciar ese proceso fue bastante complejo, pues venía de la tranquilidad del campo y el ambiente tanto de la ciudad como de la universidad me intimidó bastante. Fueron muchas las ocasiones que pensé regresar a la casa de mis papás en el campo.

Cuando estaba en los últimos semestres de la carrera me hice amiga de una muchacha que estudiaba Licenciatura en Ciencias Sociales y con ella comencé a leer libros y documentos de Educación Popular, en especial la Pedagogía el oprimido de Paulo Freire. Este libro me gustó bastante porque mostraba otro tipo de relaciones entre el educador, educando y la comunidad. Por primera vez pude sentir la pasión del autor, al mostrar la educación como el camino para ayudar a disminuir las desigualdades sociales y el empoderamiento de los sectores excluidos. De esta manera pude considerar al campo, en especial mi vereda, como un sector excluido que no era tenido en cuenta sino en época electoral. Atendiendo a ello, decidí hacer mi práctica 
docente en la escuela de la vereda en la que viven mis padres.

Pensar ahora en todas estas condiciones de vida me ha permitido abordar otras perspectivas que en su momento no consideré, por mi afán de salir de una posición que sentía era relegada y marginal. Luego de hablar con una compañera sobre la pregunta que surgió a partir de la tutoría con la profesora, comencé a pensar que ese "mejor futuro", sobre todo en el aspecto económico, que abordaba en uno de mis escritos, no necesariamente se da si las personas obtienen excelentes resultados en las pruebas y pueden acceder a la universidad; tampoco necesitan salir del campo para tener mejores oportunidades de vida, puesto que las desigualdades que hay en el campo también existen en la ciudad.

De pronto me di cuenta que aquello que he criticado, de cierta manera, hace parte de mí; que no se trata de que el oprimido salga de su lugar sin ser consciente de lo que esa posición ha configurado en él; que es necesario romper con ese binarismo para entender la complejidad de las condiciones de vida actuales, en medio de las cuales, es preciso reconocer que el sistema neoliberal influye en la manera como cada sujeto concibe su vida y su relación con el mundo. Pero llama la atención que muchas veces no logramos reconocerlo a primera vista. De esta manera, hemos ido apropiando y reproduciendo en la cotidianidad diversos discursos y formas de estar que promueven una vida en función del mercado.

Una manera de relacionarse con los demás y de construir la propia identidad. De hecho, en las sociedades denominadas como avanzadas, desde la irrupción de la producción en masa, el consumo, y especialmente el consumo de mercancías no necesarias para la supervivencia, se ha convertido en una actividad central, hasta el punto de que se puede hablar de una "sociedad consumista" (Rodríguez, 2012, pp.1-2).

De acuerdo con esta lógica de sociedad del mercado, hasta el sujeto se ha vuelto objeto y sujeto de consumo, lo que lo relega a su capacidad de compra o producción de bienes. Esta dinámica ha generado un constante deterioro en las condiciones de vida de las personas, que se evidencia en el aumento de los índices de desigualdad social a nivel mundial. Además, sostiene un claro discurso reproducido por los sistemas de mercado que ofrece la idea de progreso y éxito a través de las lógicas de consumo: entre más consumes, más tienes, más importante eres, por lo cual serás más exitoso. En ese sentido, mi formación no ha sido ajena a la influencia de estos valores promovidos por el neoliberalismo.

En mi idea de abandonar el campo, de ver el acceso al conocimiento como posibilidad de éxito, de pensar la evaluación como forma de sobresalir, hay de fondo un pensamiento influenciado por la idea del sujeto-empresa, un sujeto llamado a invertir sobre sí mismo, a adquirir conocimientos aplicables, a hacer de su vida un camino de éxito. De acuerdo con Plata (2018): 
Desde esta lógica, se considera que todos los sujetos son poseedores de un capital humano de base, definido por el conjunto de aspectos de orden cultural y cognitivo que les es propio. Pero, es precisamente este capital el que se tiene que cualificar y valorizar permanentemente si se quiere hacer parte de la dinámica o juego económico que impone la competitividad. Lo que lleva a considerar la educación como inversión en términos de los costos-beneficios que aporta (pp. 3-4).

En este punto es donde considero que la formación docente es relevante, en la medida en que puede ser una herramienta para generar prácticas pedagógicas que se contrapongan a la lógica neoliberal. Razón por la cual, un ejercicio permanente dentro de la formación política del maestro ha de ser cuestionar constantemente la configuración de nuestra propia práctica, planteándonos preguntas sobre ¿por qué educación?, ¿para qué?, ¿dónde?, ¿a quién?, ¿qué?, y ¿cómo? Preguntas que me he venido formulando en $\mathrm{mi}$ intento de hacer de mi práctica un ejercicio reflexivo.

Estos cuestionamientos y la afirmación de sentidos educativos alternativos hacen parte de un proceso de crítica como una actitud, la cual para Foucault (1995), es concebida como una manera de pensar, actuar, decir; es también una cierta relación con lo que existe, con lo que sabemos y que se consolida como una posibilidad alternativa a la explosión del arte de gobernar a los hombres. Permite cuestionar lo dado y llegar a plantearse ¿¿de qué manera estamos siendo gobernados?, y ¿cuáles son los límites del arte de gobernar?, para posteriormente declarar que no se acepta algo solo porque lo impone la autoridad, interpelando lo concerniente a las certezas y verdades. Según el autor, la crítica tiene estrecha relación con lo que Kant denomina "mayoría de edad", en cuanto es un ejercicio reflexivo y razonable que ayuda a develar las contradicciones al interior de las estructuras de poder político, económico y social, para, en ese sentido, proponer alternativas al respecto.

Como parte de este ejercicio de interrogar los modos en que se dan las actuales formas de gobierno sobre lo educativo, específicamente sobre la práctica del maestro, y al tomar como contexto mi propia práctica, fue necesario cuestionar constantemente mi quehacer como docente para poder analizar y reflexionar sobre a qué tipo de intereses responde mi propia practica educativa. En este análisis retomo a Plata (2018):

Lo primero que hay que cuestionar en las políticas y discursos oficiales es la imposición de una subjetividad externa, predeterminada, que obedece a intereses económicos, así como la empobrecida visión de lo educativo que se configura, como gestión y administración de saberes, tiempos, normas, manuales, pruebas, formatos; lo que deja por fuera lo fundante de esta relación pedagógica como acción política, o puesta al servicio de estos intereses (p. 2).

Por esta razón, al cuestionar aspectos de la formación relacionados con la 
agenda de gobierno neoliberal, es posible reconocer los procesos de subjetivación comprometidos con intereses del mundo mercantil, que, de acuerdo con las narrativas de experiencia, terminan reproduciendo los mismos discursos que sobre los que se hace la crítica; sin darnos cuenta, los apropiamos hasta el punto de pensar que son intereses propios. En las primeras narrativas de experiencia es posible reconocer en el terreno de la práctica un conflicto permanente entre el querer agenciar una educación desde lo pedagógico-político y el saberse inmerso en lógicas de captura de los sentidos colectivos, solidarios y de autorrealización bajo la forma del sujeto empresario de sí, aquel que debe esforzarse por darse una vida de éxito invirtiendo en sí mismo. Para el caso del maestro esto se traduce en tiempo dedicado a la gestión de su propia vida en función del logro de los aprendizajes de los estudiantes, lo cual implica descuidar la construcción de sentidos de vida singulares, la consecución de condiciones de vida digna. Sin embargo, los procesos de reflexividad y crítica han permitido reconocer dichos aspectos y comprender que:

Somos humanamente diferentes, irrepetibles, pero venimos de historias compartidas bio-filo-epi y ontogenéticamente, lo cual nos hace comunes a la especie humana, pero se hace específica en cada uno de acuerdo a contextos, culturas, historias, intereses, visibles en los lenguajes y habilidades que desarrollemos desde donde construimos nuestra subjetividad, que siempre está rehaciéndose (Mejía, 2020, p. 46).

Los procesos educativos requieren de un constante cuestionamiento por parte de los docentes sobre su labor, pero sobre todo, un cuestionamiento a sí mismos, un reconocerse desde las diferencias y particularidades de cada uno o en la "relación consigo mismo, como arte de vivir en función de la desujeción, para constituirse a sí mismo como un ser distinto de lo que ha dado a pensar, en orden a otra forma de pensamiento, otro modo ser y actuar" (Plata, 2018, p. 8). Entonces, se requiere de una reflexión que permita entender las prácticas educativas particulares y, sobre todo, que nos ayude a construir un sentido de vida a partir de las relaciones y prácticas que desarrollamos a diario.

Teniendo en cuanta esto, he comenzado a releer los planteamientos sobre la educación popular y crítica para entender de otra manera lo educativo, para abordar una perspectiva en la cual los contenidos no son lo primordial, sino que se da mayor importancia a los sujetos y los contextos. Atendiendo a esto y sumado a la situación de pandemia que enfrentamos, la cual ha obligado a realizar las practicas pedagógicas desde casa, he ido acercándome cada vez más a los padres de familia, lo que no siempre era posible en la presencialidad. De esta manera, he notado que en medio de la virtualidad, la familia ha podido estar más presente en los procesos educativos de los estudiantes, lo que, además, nos ha permitido trabajar a partir de los intereses de los alumnos que se han ido articulando con el contexto. Fruto de ello, ha sido posible mantener una constante realimentación entre estudiantes, docente y en este caso, madres de familia. 
Analizar estas situaciones me ha hecho entender la importancia de los procesos formativos, pero esta vez no enfocados a los estudiantes, sino los míos. Todo comienza con la necesidad de revisar y analizar a diario mis prácticas pedagógicas y, a partir de estas reflexiones, continuar con mi formación, de tal manera que pueda sentirme cada vez más orgullosa del trabajo que realizo, de mí misma y de lo que he logrado.

\section{Discusiones y Conclusiones}

De acuerdo con lo expuesto hasta el momento, la narrativa se convierte en un hecho fundamental dentro de los procesos formativos porque permite reflexionar sobre un aspecto específico y, a través de la reelaboración de los relatos, posibilita reconocer otros elementos. Los cuales, quizá no se evidencian en un primer momento, pero, se encuentran presentes en la formación y adquieren importancia gracias a las reflexiones y nuevas percepciones que surgen mientras se avanza en el proceso de escritura.

En los procesos narrativos es importante el papel que cumple la experiencia, puesto que, al volver sobre los relatos, las percepciones de un acontecimiento específico van cambiando o se van resignificando. Esto permite que tal experiencia adquiera otro significado en la medida en que va siendo reelaborada. De esta manera, las prácticas formativas se trasforman teniendo en cuenta que las experiencias cambian o se modifican.

El reconocimiento de lo político en el cuidado de la vida es una de las categorías que surgió al realizar y analizar los relatos, con lo cual se reconocen las prácticas de cuidados, de sí mismo y de los demás, como indispensables dentro de nuestra cotidianidad. Estas prácticas se consideran imprescindibles dentro del campo de la educación, por tanto, generan grandes desafíos a nivel de nuestras relaciones en comunidad.

\section{Referencias}

Africano, M. (2018). Configuración de la subjetividad del maestro, concepción y sentido de su labor, el caso de dos colegios de Bogotá [Tesis de maestría, Universidad Pedagógica Nacional]. Repositorio Institucional UPN. http://hdl.handle. net $/ 20.500 .12209 / 10380$

Castro, C. (2018). Desde la acción política feminista: instituciones y sociedad civil organizada transformando realidades. Economistas sin Fronteras, (29), 11-15. http://ecosfron.org/wp-content/uploads/Dossier-29_finales.pdf

Passeggi, M. (2011). Narrativas Autobiográficas: solidaridad yética en educación. Rizoma freireano, 11, 1-11. http://www.rizoma-freireano.org/articles-1111/narrativasautobiograficas-solidaridad-y-etica-en-educacion-maria-da-conceicao-passeggi

Foucault, M. (1994). Hermenéutica del sujeto (F. Álvarez-Uría, trad.). Ediciones de la Piqueta. 
Foucault, M. (1995). ¿Qué es la crítica? Daimon Revista Internacional de Filosofia, 11, 5-26. https://revistas.um.es/daimon/article/view/7261

Itaí, M. (2012). La formación de la subjetividad política. Revista colombiana de educación, (63), 321-328. https://doi.org/10.17227/01203916.1705

Larrosa, J. (2006). Sobre la experiencia. Revista Educación y Pedagogía, 18, 52-67. https://revistas.udea.edu.co/index.php/revistaeyp/article/view/19066

Mejía, M. (2020). Educación(es), escuela (s) y pedagogía(s) en la cuarta revolución industrial desde Nuestra América. Ediciones desde abajo.

Plata, M. (2018). Subjetividades docentes en tiempos de la excelencia educativa. Colombian Applied Linguistics Journal, 20(2), 275-290. https://doi. org/10.14483/22487085

Rodríguez, S. (2012). Consumismo y sociedad: una visión crítica del homo consumens. Nómadas. Revista Crítica de Ciencias Sociales y Jurídicas, 34 (2), 189-210. http://dx.doi.org/10.5209/rev_NOMA.2012.v34.n2.40739

Sáenz, M. (2018). Repensar el mundo desde los cuidados. Economistas sin Fronteras, (29), 16-20. http://ecosfron.org/wp-content/uploads/Dossier-29_finales.pdf 